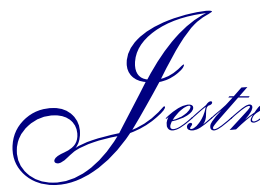

Journal of Engineering Science and Technology Review 8 (5) (2015) 41-48

JOURNAL OF

Engineering Science and

Technology Review

Review Article

www.jestr.org

\title{
A Review of Image Denoising Methods
}

\author{
I. Irum*, M. A. Shahid, M. Sharif and M. Raza \\ COMSATS Institute of Information Technology Wah Cantt, Pakistan
}

Received 10 August 2015; Accepted 19 December 2015

\begin{abstract}
Image Denoising is one of the fundamental and very important necessary processes in image processing. It is still a challenging and a hot problem for researchers. Images are one of essential representations in every field like education, agriculture, geosciences, aerospace, surveillance, entertainment etc by means of electronic or print media. Images can get corrupted by noise, there has been a great research effort which made solutions for this problem, a number of methods have been proposed. An overview of various methods is given here after a brief introduction. These methods have been categorized on the bases of techniques used.
\end{abstract}

Keywords: Derivative Based Denoising, Fuzzy Based Denoising, Mathematical Morphology Based Denoising, Median Based Denoising, Nonlinear Denoising methods, s Statistical Modeling Based Denoising

\section{Introduction}

Various sources let the digital images to be corrupted by poor contrast and noise, theses sources include image transmission, acquisition, compression [1-5], quantization, illumination conditions [6], malfunctioning instruments, ill positions etc. These sources directly degrade the visual quality of image during processing of image [7]. Process of image denoising or image restoration is targeted to estimate the original image from corrupted image. It is still the most fundamental, largely unsolved and widely studied problem [8]. Image has important information and certain details, as communication through visual images is an integral part of modern life. Images and videos are used everywhere to communicate as a visual source. Noise affects image features seriously like edges, thin lines, and loss of image details provides degradation of spatial resolutions. Therefore noise removal from corrupted images is very important and necessary before further processing on them like segmentation [9-12], feature matching, edge detection, feature extraction [13-15], feature detection [16] of image details used for face recognition [17-38] etc, These denoised images can be used for face detection [39-41], content based image retrieval [42-48], medical image reconstruction [49], understanding Morphology of medical images [50] with its applications [51], medical image enhancement [52], image rendering [53] etc.

A huge number of methods have been proposed in this context over the past decades in image processing. A review of these methods has been given here covering the representative methods which gave better performance in this area. These methods have been categorized on the bases of their natures of techniques used in. These categories are

* E-mail address: ismairum@gmail.com ISSN: 1791-2377 (c) 2015 Kavala Institute of Technology. All rights reserved.
Median Based, Statistical Modeling Based, Derivative Based, Fuzzy Logic Based and Mathematical Morphology Based image denoising methods. These categories are discussed one by one in upcoming section of rest of the paper and conclusion is given at the end.

\section{Median Based Image Denoising Methods}

Median Based Filters or Denoising Methods are the corner stones of image cancellation methods in modern image processing. Tukey [54] first introduced the Median Filter, after its inception, tremendous efforts have been made for optimization, improvement and refinement over the years. Standard median filters presented in [55] treated all the pixels of the image whether corrupted or uncorrupted. [56] To overcome this drawback Weighted Median Filters (WMF) [57] and Switching Median Filter (SMF) [58] were proposed. WMF reduced the smoothing effect, preserved the image sharpness and treated the entire pixels like standard median filter by giving higher weights to the central pixel [59-60]. Weighted Order Statistics Median Filters (WOSFs) were proposed in [61]. Design of WMF admitting negative and complex weights presented in [62-65]. Steerability concept was introduced in [66] and its application found in [67]. Recently Dimitrios Charalampidis [68] proposed steerable WMF inheriting the noise robustness and edge preserving capabilities of WMF.

SMF reduced the number of pixels subjected to filtration by identifying the corrupted and uncorrupted pixels and leaving the uncorrupted pixels unchanged. In [69-80] extensions of median filter and SMF have been presented. Recent examples of noise adaptive approaches can be found in [81-83]. The parameters that can be used for as input function to adaptive approach can be window size, shape or rank [84]. It created blurred images when applied to mix or Gaussian Noise, Directional Weighted Median (DWM) [85] 
presented an iterative filtering approach to overcome this drawback. Hsing [86] proposed switching bilateral filter based on median based detection scheme and to address the problems presented in [87]. Partition based techniques comprising linear combination; made with current pixel value and output of nonlinear median filter has been studied in [88]. Some hybrid techniques of linear and non linear filters can be investigated in [89]. Wang and $\mathrm{Lu}$ [90] proposed LOFBDND an efficient SMF based on combination of Local Outlier Factor (LOF) [91], Boundary Discriminative Noise Detection (BDND) and Directional Weighted Median Filter.

\section{Statistical Modeling Based Image Denoising}

Due the sparseness property of multiresolution image representation in non-Gaussian statistics for wavelet coefficients, multiresolution image representation gained much attention for probabilistic modeling over the last decade. Moreover, statistical characteristics of image are accurately described in multiresolution and sparse domains like, Discrete Wavelet Transform (DWT) [92], Discrete Complex Wavelet Transform (DCWT) [93], Discrete Contourlet Transform (DCT) [94] and Discrete Curvelet Transform (DCUT) [95]. Sparse domain image denoising consists of three steps (a) transformation of noisy observation into signal (b) estimation of image from transformed coefficients (c) synthesis step that inverse transformation of selected coefficients [96]. Image estimation functions from the transformed coefficients are of multiple types for example hard and soft thresholding [97], firm and garrote thresholding [98]. Marginal histograms of wavelet coefficients are of two characteristics leptokurtic peaked and heavy tailed [99]. Some of image denoising methods are seemed to exploit these characteristics of marginal histograms of wavelet coefficients and follow simple and appropriate shrinkage rules [100-115]. Another characteristic of wavelet coefficients is interscale dependency. This property is characterized by local parameters of probability density function. This is observed that algorithms which use local parameters as outperform as compared with algorithms using global parameters [116124]. Study of joint histograms of wavelet coefficients has been given in [125-129] additionally.

Recent variations of Gaussian scale mixture (GSM) have been presented in [130-132]. Some statistical models of wavelet coefficients estimation in sparse domain have been presented in [133-139].

\section{Derivative Based Image Denoising}

There has been a wide use of partial differential equations in edge preservation image denoising over the past decade. These methods can be of two types, nonlinear diffusion and energy functional minimization, the nonlinear diffusion is axiomatic approach of nonlinear scale space and energy functional minimization is variational approach. There has been a large number of PDE models have been presented over the past years; some of representative models are being described here.

\subsection{Axiomatic approach}

Witkin [140] proposed the original PDE model based on linear heat equation diffusing in all direction and destroying the edges. This limitation enlightened the new ways for researchers like controlling diffusion speed, direction, adding a fidelity term or combination of these. Perona-Malik [141] were pioneers for introducing such scheme by controlling the diffusion speed and proposing a nonlinear adaptive diffusion process named anisotropic diffusion. Catte [142], You and Kaveh [143] proposed PDE image denoising models based on improving controlling speed function by a selective smoothing model and by using Laplacian image of evolving image instead of gradient image respectively. Black [144] proposed new controlling function based on statistical interpretation of anisotropic diffusion called edge stopping function, Chen [145] introduced coupled nonlinear diffusion model in which the controlling function is smoothed by diffusion equation. Sapiro and Ringach [146] used the combination of speed and direction of diffusion for the improvement of controlling function, Tschumperlé and Deriche [147] introduced a unifying expression for vector valued images to regularize PDE to achieve desired smoothing behaviors by summarizing the existing PDE based formalisms. Vosburg [148] proposed speckle noise removal approach from ultrasonic images based on study of numerical scheme of anisotropic diffusion filter and extending it to matrix of anisotropic diffusion. Wang and Zhang [149] used the local variance to control the forward and backward diffusion.

\subsection{Variation approach}

Rudin [150] introduced Total Variation Denoising (TVD) model by giving the fidelity term a simple form and defining it as dynamic value. [151] set the fidelity term as a constant value and used nonconvex functions as fidelity functions. Nikolova [152] described the usage of nonconvex functions to decrease the goodness of fit to the contaminated data in case of very high noise density to stabilize the denoising Processing Abubakar [153] proposed multiplicative regularization scheme for TV deblurring problems, in which regularization parameter is controlled by an optimization Processing Gilboa [154] presented the pyramidal texture structure-texture decomposition and analysis for preservation of texture and certain image details. Spatially varying fidelity term controlled the denoising process in image regions. Wang [155] combined the pixel and wavelet domain where TV and shrinkage are used as regularizers in both domains respectively. Chartrand [156] presented quasi Newton method for TV regularization. Bae [157] presented the TV minimization based on graph cuts method. Wu and Tang [158] distinguished the pixels as edge, noise and interior pixels, and on bases of these definition defined the speed function and fidelity term addressing the limitations of impulse noise removal, that existing approaches treated corrupted and un corrupted pixels in same way, in case of high noise densities and less iterations left the corrupted pixels. Zuo [159] proposed generalized accelerated approximation gradient (GAPG) algorithm concerned with TV based image restoration problems

\subsection{Complex diffusion processes}

Gilboa [160] proposed regularized shock filter and ramp preserving denoising process by complex diffusion process by using the free Schrödinger equation. Some other examples of complex diffusion can be seen in [161-162]

\section{Fuzzy Logic based Image Denoising}

Since their inception neural networks and fuzzy systems are capable of learning from examples and to deal with the 
uncertainty respectively. Image denoising is surly a filtering system with visible fuzziness as the existence of fuzziness in the image signal and contaminated signal. Hence fuzzy systems have achieved great focus of research efforts recently. Russo [163] has reviewed the applications, filters and advancement of fuzzy systems covering classical methods [164], fuzzy weight filters [165] and fuzzified FIRE filters [166]. Zhang [167] proposed fuzzy based impulse noise detection and removal by using long-range correlation among different image portions. Examples of growing fuzzy systems applications are presented in [168-170]. Neurofuzzy systems developed by combining the neural network and fuzzy systems have also been used for image denoising [172]. Lee [171] proposed a fuzzy filter using genetic learning process, Yuksel [172] combined median filter, edge detector and neurofuzzy network to develop hybrid filter in which internal parameters adaptive optimization process is done with training. Schulte and others in [173-176] proposed fuzzy derivative estimation and fuzzy smoothing for noise detection and removal respectively. These methods perform better but with more memory and computation cost. Liang [177] proposed two stage neurofuzzy techniques for impulse noise removal. Toh and Isa [178-179] dealt with uncertainties present in local information with fuzzy reasoning and proposed Cluster-based Adaptive Fuzzy Switching Median (CAFSM) to remove all kinds of noise and salt and pepper respectively. Type-2 fuzzy logic systems (FLSs) have been getting great interest in applications in recent years [180-183]. Membership functions of type-1 FLSs are scalar whereas of type-2 FLSs these functions are fuzzy. This double degree of fuzziness makes type-2 FLSs more efficient. Fuzzy logic based systems have shown promising results due to the uncertainty in image data and fuzzy nature of image filtering.

\section{Mathematical Morphology Based Image Denoising}

Mathematical morphology is about shapes and structures in image processing, it is a geometrical approach with strong mathematical analysis. Mathematical morphology based image denoising has been attracting researchers recently. The research efforts are being given to design a nonlinear operator to extract geometric and topological information from images.

Mathematical morphology is application related to lattice theory to spatial structures. Adaptive mathematical morphology [184-186] is the morphological techniques having the capability of adapting the local context of the signal. Some kinds of morphological filters present are multi structure elements based morphological filter [187-188], soft morphological filters [189-191] and hybrid operator based morphological filters [192]. These approaches improved the performance of image denoising. Morphological dual operators [193-194] are key techniques to construct morphological hybrid filters.

\section{Conclusion}

We have focused on several methods of image denoising in nonlinear domain, although the linear methods are simple, effective and easy to implement but they are limited with high noise densities and complex noise models. Nonlinear methods have been the state of art denoising methods. The methods differ from each other on the type of noise used. All types of noise for example impulse, Gaussian white noise and speckle have been kept under considerations with their associated denoising methods. Every method has its own performance measures in its problem domain which may not work and fulfill the requirements in other problem domain. However, overall methods associated with wavelet domain have achieved great performance due to their noise adaptive and sparseness. Median based methods have been outstanding for image restoration because of nonlinearity but comparatively spatial domain computations are complex and time consuming. Fuzzy logic based methods have shown promising results.

\section{References}

1. Mehwish Rehman, Muhammad Sharif, Mudassar Raza, Image Compression-A Survey, Journal of Applied Sciences, Engineering and Technology, 7(4): 656-672, 2014.

2. Saleha Masood, Muhammad Sharif, Mussarat Yasmin, Mudassar Raza, Sajjad Mohsin, "Brain image Compression: A brief survey", Research Journal of Applied Sciences, Engineering and Technology, Vol. 5, pp. 49-59, 2013.

3. Mudassar Raza, Ahmed Adnan, Muhammad Sharif, Syed Waqas Haider, "Lossless Compression Method for Medical Image Sequences Using Super-Spatial Structure Prediction and Interframe Coding", Journal of Applied Research and Technology (JART) Vol. 10 No. 4, August 2012

4. Qaiser Naeem, Muhammad Sharif, Mudassar Raza," Improving Audio Data Quality and Compression", Emerging Technologies, 2008. ICET 2008. 4th International Conference on, 2008, pp. 332$337,2008$.

5. Rana Muhammad Nazim, Muhammad Sharif, Mudassar Raza, Aman Ullah Khan "Layered Compression Technique (LCT) Based on Entropy or Dictionary Methods", The First International Conference on Computer, Control \& Communication (IC4), organized by Pakistan Navy Engineering College (PNEC), a constituent college of National University of Sciences \& Technology (NUST), in collaboration with IEEE Karachi Sector, Higher Education Commission (HEC) and Pakistan Council of Scientific and Industrial Research (PCSIR), 12-13 November, 2007 at PNEC, Karachi, Pakistan, 2007
6. Muhammad Sharif, Sajjad Mohsin, Muhammad Jawad Jamal, Mudassar Raza, Illumination Normalization Preprocessing for face recognition", in Environmental Science and Information Application Technology (ESIAT), 2010 International Conference on, 2010, pp. 44-47, 2010

7. N. Damera-Venkata, T. D. Kite, W. S. Geisler, B. L. Evans, and A. C. Bovik, Image quality assessment based on a degradation model, IEEE Transaction on Image Processing, vol. 9, no. 4, pp. 636-650, April,2000

8. A. Buades, B. Coll, and J. M. Morel, On Image Denoising Methods, http://www.cmla.ens-cachan.fr/Cmla/, (2004). To appear in SIAM Multiscale Modeling and Simulation, CMLA Preprint, 2004

9. M. Hameed, M. Sharif, M. Raza, S. W. Haider, and M. Iqbal, "Framework for the Comparison of Classifiers for Medical Image Segmentation with Transform and Moment based features", Research Journal of Recent Sciences, Vol. 2, pp. 1-10, 2013.

10. W. Haider, M. Sharif, and M. Raza, "Achieving Accuracy in Early Stage Tumor Identification Systems based on Image Segmentation and 3D Structure Analysis," Computer Engineering and Intelligent Systems, Vol. 2, pp. 96-102, 2011.

11. Amir Shahzad, Muhammad Sharif, Mudassar Raza, Khalid Hussain, "Enhanced Watershed Image Processing Segmentation", Volume 2 - issue 1 Journal of Information and Communication Technology Vol. 2, No. 1, (Spring 2008) 01-09, 2008 
12. Yasmin, Mussarat, Muhammad Sharif, Sajjad Mohsin, and Faisal Azam. "Pathological Brain Image Segmentation and Classification: A Survey." Current Medical Imaging Reviews 10, no. 3 (2014): 163-177.

13. Asma Shaheen, Syed Muhammad Anwar and Muhammad Sharif," Feature Extraction and Classification of Epilepsy in Different Seizure Types: A Survey", Current Medical Imaging Reviews, Volume 10, issue 2, Pp: $77-83,2014$

14. M. Sharif, M. Raza, S. Mohsin, and J. H. Shah, "Microscopic Feature Extraction Method", Int. J. Advanced Networking and Applications, Vol. 4, pp. 1700-1703, 2013.

15. Azeem, A., M. Sharif, J. H. Shah, and M. Raza. "Hexagonal scale invariant feature transform (H-SIFT)for facial feature extraction" Journal of Applied Research and Technology 13 (2015): 402-408.

16. Muhammad Sharif, Jamal Hussain Shah, Sajjad Mohsin and Mudassar Raza, "Facial Feature Detection and Recognition for Varying Poses", World Congress on Engineering and Computer Science 2014 (International Conference on Signal Processing and Imaging Engineering 2014), 22-24 October, 2014, San Francisco, USA, 2014

17. Marryam Murtaza, Muhammad Sharif, Mudassar Raza, Jamal Hussain Shah, "Face Recognition Using Adaptive Margin Fisher's Criterion Linear Discriminant Analysis (AMFC-LDA)", The International Arab Journal of Information Technology (IAJIT), Volume 11, No. 2, March 2014

18. Muhammad Sharif, Muhammad Atif Ali, Mudassar Raza, Sajjad Mohsin, "Face Recognition using Edge Information and DCT", Sindh University Research Journal (SURJ) (SCIENCE SERIES), Volume 43 (2) 209-214, 2011

19. Muhammad Sharif, Sajjad Mohsin and Muhammad Younas Javed," A Survey: Face Recognition Techniques", Research Journal of Applied Sciences, Engineering and Technology, Vol. 4. No. 23 pp 4979-4990, 2012

20. Muhammad Sharif, Adeel Khalid, Mudassar Raza, Sajjad Mohsin, "Face Recognition Using Gabor Filters", Journal of Applied Computer Science \& Mathematics, Volume 5, issue 11, pp 53-57, 2011

21. Aisha Azeem, Muhammad Sharif, Mudassar Raza, Marryam Murtaza, "A Survey: Face Recognition Techniques Under Partial Occlusion", The International Arab Journal of Information Technology (IAJIT) (Impact Factor) (will be published on Volume 11, No. 1, January 2014), 2014

22. Shah, Jamal Hussain, Muhammad Sharif, Mudassar Raza, and Aisha Azeem. "Face recognition across pose variation and the $3 \mathrm{~S}$ problem." Turkish Journal of Electrical Engineering and Computer Science 22, no. 6 (2014): 1423-1436.

23. Jamal Shah, Muhammad Sharif, Mudassar Raza, Aisha Azeem, "A Survey: Linear and Nonlinear PCA Based Face Recognition Techniques", The International Arab Journal of Information Technology (IAJIT) Volume 10, No. 6, November 2013.

24. Muhammad Sharif, Jamal H. Shah, Sajjad Mohsin, and Mudassar Raza,"Sub-Holistic Hidden Markov Model for Face Recognition", Research Journal of Recent Sciences, Vol. 2, pp. 10-14, 2013.

25. Muhammad Sharif, Adeel Khalid, Mudassar Raza, Sajjad Mohsin, "Face Detection and Recognition Through Hexagonal Image Processing”, Sindh Univ. Res. Jour. (Sci. Ser.) Vol.44 (3) 359-366, 2012

26. Muhammad Sharif, Sajjad Mohsin, Saad Anis, Mudassar Raza, "Enhanced SVD Based Face Recognition", Journal of Applied Computer Science \& Mathematics, issue 12(6), 2012

27. Muhammad Sharif, Muhammad Younas Javed, Sajjad Mohsin, "Face Recognition Based on Facial Features", Research Journal of Applied Sciences, Engineering and Technology 4(17): 2879-2886, 2012

28. Muhammad Sharif, Sajjad Mohsin, Muhammad Younas Javed, and Muhammad Atif Ali," Single Image Face Recognition Using Laplacian of Gaussian and Discrete Cosine Transforms" the International Arab Journal of Information Technology (IAJIT), 9, no. 6 (2012): 562-570, 2012

29. Muhammad Sharif, Kamran Ayub, Danish Sattar, Mudassar Raza, Sajjad Mohsin," Enhanced and Fast Face Recognition by Proposing Hashing Algorithm", Journal of Applied Research and Technology (JART), Vol. 10, pp. 607-617, 2012.

30. Muhammad Sharif, Sajjad Mohsin, Muhammad Jawad Jamal, Muhammad Younas Javed, Mudassar Raza, "Face Recognition for Disguised Variations Using Gabor Feature Extraction" Australian Journal of Basic and Applied Sciences, Vol. 5, pp. 1648-1656, 2011.

31. Muhammad Sharif, Sajjad Mohsin, Rana Abdul Hanan, Muhammad Vounas Iaved Mudacsar Raza "I Tsino Noce
Heuristics for Efficient Face Recognition", Sindh University Research Journal (SURJ) (SCIENCE SERIES) Vol.43 (1-A) 63-68, 2011

32. Muhammad Sharif, Muhammad Kamran Ayub, Mudassar Raza and Sajjad Mohsin," Data Reductionality Technique for Face Recognition", Proceedings of the Pakistan Academy of Sciences, 48 (4): 229-234, 2011

33. Muhammad Sharif, Sajjad Mohsin, Rana Abdul Hanan, Muhammad Younas Javed, Mudassar Raza, "3d Face Recognition Using Horizontal And Vertical Marked Strips", Sindh University Research Journal (SURJ) 2011 Volume 43 No.01-A JUNE http://www.usindh.edu.pk/surj/261-vol-43-1a-2011

34. Muhammad Sharif, Sajjad Mohsin, Muhammad Jawad Jamal, Mudassar Raza, "Illumination Normalization Preprocessing for face recognition", in Environmental Science and Information Application Technology (ESIAT), 2010 International Conference on, 2010, pp. 44-47

35. Aisha Azeem, Muhammad Sharif, Mudassar Raza, Marryam Murtaza, "A Survey: Face Recognition Techniques Under Partial Occlusion", The International Arab Journal of Information Technology (IAJIT) (Impact Factor) (will be published on Volume 11, No. 1, January 2014)

36. Marryam Murtaza, Muhammad Sharif, Mudassar Raza, Jamal Hussain Shah, "Analysis of Face Recognition under Varying Facial Expression: A Survey", The International Arab Journal of Information Technology (IAJIT) Volume 10, No.4, July 2013

37. Shah, Jamal Hussain, Muhammad Sharif, Mudassar Raza, and Marryam Murtaza. "Robust Face Recognition Technique under Varying Illumination." Journal of Applied Research and Technology 13, no. 1 (2015): 97-105.

38. Muhammad Sharif, Sajjad Mohsin and Muhammad Younas Javed," A Survey: Face Recognition Techniques", Research Journal of Applied Sciences, Engineering and Technology, Vol. 4. No. 23 pp 4979-4990, 2012

39. Muhammad Sharif, Sajjad Mohsin, Muhammad Younas Javed," Real Time Face Detection Using Skin Detection (Block Approach)", Journal of Applied Computer Science \& Mathematics, no. 10 (5) 2011

40. Muhammad Sharif, Muhammad Kamran Ayub, Danish Sattar and Mudassar Raza," Real Time Face Detection", Sindh University Research Journal (Science Series), Vol.44 (4) 597-600, 2012

41. Muhammad Sharif, Sajjad Mohsin, Muhammad Younas Javed," Real Time Face Detection Using Skin Detection (Block Approach)", Journal of Applied Computer Science \& Mathematics, no. 10 (5) 2011

42. Yasmin Mussarat, Muhammad Sharif, Sajjad Mohsin, Isma Irum, "Content Based Image Retrieval using Combined Features of Shape, Color and Relevance Feedback", KSII Transactions on Internet and Information Systems, 7(12), 2013

43. Yasmin, Mussarat, Sajjad Mohsin, Isma Irum, and Muhammad Sharif. "Content Based Image Retrieval by Shape, Color and Relevance Feedback", Life Science Journal 10, no. 4s, 2013.

44. M. Rehman, M. Iqbal, M. Sharif, and M. Raza, "Content Based Image Retrieval: Survey," World Applied Sciences Journal, Vol. 19, pp. 404-412, 2012.

45. Yasmin Mussarat, Mohsin Sajjad, Sharif Muhammad, "Use of Low Level Features for Content Based Image Retrieval Survey", Research Journal of Recent Sciences, Vol. 2(11), November (2013)

46. Mussarat Yasmin, Sajjad Mohsin, Muhammad Sharif," Intelligent Image Retrieval Techniques: A Survey", Journal of Applied Research and Technology Volume 12, No.1 December 2013

47. Yasmin, Mussarat, Muhammad Sharif, and Sajjad Mohsin. "IMAGE RETRIEVAL TECHNIQUES USING SHAPES OF OBJECTS: A SURVEY." Sci.Int (Lahore),25(4),723-729,2013

48. Mussarat Yasmin, Muhammad Sharif, Isma Irum and Sajjad Mohsin, "An Efficient Content Based Image Retrieval using EI Classification and Color Features" Journal of Applied Research and Technology (JART) 2014

49. Mussarat Yasmin, Sajjad Mohsin, Muhammad Sharif, Saleha Masood, Mudassar Raza , "Brain Image Reconstruction, A Short Survey”, World Applied Sciences Journal (WASJ) 19 (1): 52-62, 2012

50. I. Irum, M. Raza, and M. Sharif, "Morphological Techniques for Medical Images: A Review," Research Journal of Applied Sciences, Engineering and Technology, Vol. 4, pp. 2948-2962, 2012.

51. Yasmin, Mussarat, Muhammad Sharif, and Sajjad Mohsin. "Neural Networks in Medical Imaging Applications: A Survey", World Applied Sciences Journal 22, no. 1 (2013): 85-96. 
52. Mussarat Yasmin, Muhammad Sharif, Saleha Masood, Mudassar Raza, and S. Mohsin, "Brain Image Enhancement-A Survey," World Applied Sciences Journal, vol. 17, pp. 1192-1204, 2012

53. Mudassar Raza, Muhammad Sharif, Mussarat Yasmin, Saleha Masood, Sajjad Mohsin, "Brain Image Representation and Rendering: A Survey", Research Journal of Applied Sciences, Engineering and Technology 4(18): 3274-3282, 2012

54. Tukey J.W, Exploratory Data Analysis Reading, MA. Addison Wesly, 1977

55. Astola. J and Kuosmanen. P, Fundamentals of nonlinear digital filtering, CRC Press, 1997

56. Guangin. $Z$ and Jiegu. L, Some problems of 2D morphological and median filters, J. Shanghai Univ. (English Edition), 1, (3), pp. 245248, 1997

57. Yin. L, Yang. R, Gabbouj. $M$ and Neuvo. Y, Weighted median filters: a tutorial, IEEE Transactions on Circuits Systems, 43, (3), pp. $157-192,1996$

58. D. Florencio and R. Schafer, Decision-based median filter using local signal statistics, in Proceedings SPIE International Symposium on Visual Communications and Image Processing, Chicago, September, 1994

59. Chen. T, Wu and H.R, Adaptive impulse detection using centerweighted median filters, IEEE Signal Processing Letters, 8, (1), pp. $1-3,2001$

60. Chan, R.H, Chen Hu Nikolova and M, An iterative procedure for removing random-valued impulse noise, IEEE Signal Processing Letters, 11, (12), pp. 921-924, 2004

61. P.-T. Yu and W.-H. Lao, Weighted order statistics filter-Their classification, some properties, and conversion algorithm, IEEE Transactions on Signal Processing, vol. 42, no. 10, pp. 2483-2497, October, 1994

62. K.-S. Choi, A. W. Morales, and S.-J.Ko, Design of linear combination of weighted medians, IEEE Transactions on Signal Processing, vol. 49, no. 9, pp. 1940-1952, September, 2001

63. I. Shmulevich and G. R. Arce, Spectral design of weighted median filters admitting negative weights, IEEE Signal Processing Letters, vol. 8, no. 12, pp. 313-316, December, 2001

64. G. R. Arce, Nonlinear Signal Processing: A Statistical Approach, New York: Wiley, November, 2004

65. S. Hoyos, Y. Li, J. Bacca, and G. R. Arce, Weighted median filters admitting complex-valued weights and their optimization, IEEE Transactions on Signal Processing, vol. 52, no. 10, pp. 2776-2787, Part 1, October, 2004

66. W. T. Freeman and E. H. Andelson, The design and use of steerable filters, IEEE Transactions on Pattern Analysis and Machine Intelligence, vol. 13, no. 9, pp. 891-906, September, 1991

67. A. A. Bharath and J. Ng, A steerable complex wavelet construction and its application to image denoising, IEEE Transactions on Image Processing, vol. 14, no. 7, pp. 948-959, July, 2005

68. Dimitrios Charalampidis, Steerable Weighted Median Filters, IEEE Transactions on Image Processing, Vol. 19, No. 4, April, 2010

69. T. Chen and H. R. Wu, Space variant median filters for the restoration of impulse noise corrupted images, IEEE Transactions on Circuits System II, Analog Digital Signal Processing, vol. 48, no. 8, pp. 784-789, August, 2001

70. P. S. Windyga, Fast impulsive noise removal, IEEE Transactions on Image Processing, vol. 10, no. 1, pp. 173-179, January, 2001

71. G. Pok, J. C. Liu, and A. S. Nair, Selective removal of impulse noise based on homogeneity level information, IEEE Transactions on Image Processing, vol. 12, no. 1, pp. 85-91, January, 2003

72. N. Alajlan, M. Kamel, and E. Jernigan, Detail preserving impulsive noise removal, Signal Processing: Image Communication, vol. 19, pp. 993-1003, 2004

73. H. Xu, G. Zhu, F. Peng, and D.Wang, Adaptive fuzzy switching filter for images corrupted by impulse noise, Pattern Recognition Letters, vol. 25, pp. 1657-1663, 2004

74. V. Crnojevic, Impulse noise filter with adaptive MAD-based threshold, in Proceedings International Conference on Image Processing, pp. 337-340, March, 2005

75. R. H. Chan, C. W. Ho, and M. Nikolova, "Salt-and-pepper noise removal by median-type noise detectors and detail preserving regularization," IEEE Transactions on Image Processing, vol. 14, no. 10, pp. 1479-1485, October, 2005

76. P. E. Ng and K. K. Ma, A switching median filter with boundary discriminative noise detection for extremely corrupted images, IEEE Transactions on Image Processing, vol. 15, no. 6, pp. 15061516, June, 2006
77. K. S. Srinivasan and D. Ebenezer, A new fast and efficient decision based algorithm for removal of high-density impulse noises, IEEE Signal Processing Letters vol. 14, no. 3, pp. 189-192, March, 2007

78. K. Aiswarya, V. Jayaraj, and D. Ebenezer, A new and efficient algorithm for the removal of high density salt and pepper noise in images and videos, in Second International Conference Computer Modeling and Simulation, 2010

79. S. Esakkirajan, T. Veerakumar, Adabala N. Subramanyam, and C. H. PremChand, Removal of High Density Salt and Pepper Noise Through Modified Decision Based Unsymmetric Trimmed Median Filter, IEEE Signal Processing Letters, Vol. 18, No. 5, May, 2011

80. T. Chen and H. R. Wu, Impulse noise removal by multi-state median filtering, in Proceedings IEEE International Conference on Acoustics, Speech, Signal Processing, vol. IV, June (2000), pp. 2183-2186, 2000.

81. A. Fabijan and ska D. Sankowski, "Noise adaptive switching median-based filter for impulse noise removal from extremely corrupted images, IET Image Processing, Vol. 5, Iss. 5, pp. 472480, 2011

82. S. Liu, Adaptive scalar and vector median filtering of noisy colour images based on noise estimation, IET Image Processing, Vol. 5, Iss. 6, pp. 541-553, 2011

83. K. Tripathi, U. Ghanekar and S. Mukhopadhyay, Switching median filter: advanced boundary discriminative noise detection algorithm, IET Image Processing, Vol. 5, Iss. 7, pp. 598-610, 2011

84. How-Lung Eng and Kai-Kuang Ma, Noise Adaptive Soft-Switching Median Filter, IEEE Transactions On Image Processing, Vol. 10, No. 2, February, 2001

85. Y. Dong and S. Xu, A new directional weighted median filter for removal of random-valued impulse noise, IEEE Signal Processing Letters, vol. 14, no. 3, pp. 193-196, March, 2007

86. Chih-Hsing Lin, Jia-Shiuan Tsai, and Ching-Te Chiu, Switching Bilateral Filter With a Texture/Noise Detector for Universal Noise Removal, IEEE Transactions On Image Processing, Vol. 19, No. 9, September, 2010

87. R. Garnett, T. Huegerich, C. Chui, and W. He, A universal noise removal algorithm with an impulse detector, IEEE Transactions on Image Processing, vol. 14, no. 11, pp. 1747-1754, November, 2005

88. Tao Chen and Hong Ren Wu, Application of Partition-Based Median Type Filters for Suppressing Noise in Images, IEEE Transactions On Image Processing, Vol. 10, No. 6, June, 2001

89. Hakan Güray S, enel, Richard Alan Peters, II, and Benoit Dawant, Topological Median Filters, IEEE Transactions On Image Processing, Vol. 11, No. 2, February, 2002

90. Wei Wang and Peizhong Lu, An Efficient Switching Median Filter Based on Local Outlier Factor, IEEE Signal Processing Letters, Vol. 18, No. 10, October, 2011

91. M.M. Breuig, "LOF: Identifying density based local outliers[C]," in Proceedings ACM SIGMOD Conference, New York, (2000), pp. 427-438., 2000

92. Mallat S.G, A Wavelet Tour Of Signal Processing, Academic Press, San Diego, 1998

93. Selesnick I.W, Baraniuk R.G and Kingsbury N, The Dual-Tree Complex Wavelet Transforms - A Coherent Framework For Multiscale Signal And Image Processing, IEEE Signal Processing Magzine, (2005), 22, (6), Pp. 123-151, 2005

94. Candes E., Donoho D and Curvelets: A Surprisingly Effective Nonadaptive Representation Of Objects With Edges, In COHEN A., RABUT C and SCHUMAKER L.L. (EDS.): Curves And Surface' (Vanderbilt University Press, Nashville, TN, 1999), Pp. 123-143, 1999

95. Do M.N and Vetterli M, The Contourlet Transform: An Efficient Directional Multiresolution Image Representation, IEEE Transactions on Image Processing, 14, (12), Pp. 2091-2106, 2005

96. H. Rabbani and S. Gazor, Image Denoising Employing Local Mixture Models In Sparse Domains, IET Image Processing, Vol. 4, Iss. 5, Pp. 413-428, 2010

97. D. L. Donoho, De-Noising By Soft-Thresholding, IEEE Transactions on Information Theory, Vol. 41, Pp. 613-627, May, 1995

98. Gao H, Wavelet Shrinkage Denoising Using The Nonnegative Garrote, Journal of Computer Graphics and Statistics, (1998), 7, Pp. 469-488, 1998

99. S. Mallat, Multifrequency Channel Decomposition Of Images And Wavelet Models, IEEE Transactions on Acoustics, Speech, Signal Processing, Vol. 37, No. 12, Pp. 2091-2110, December, 1989

100.Chang S.G, Yu B and Vetterli M, Adaptive Wavelet Thresholding For Image Denoising And Compression, IEEE Transactions on Imace Procescino Q (9) Pn 153?-1546 900ก 
101.S. Chang, B. Yu, And M. Vetterli, Spatially Adaptive Wavelet Thresholding With Context Modeling For Image Denoising, IEEE Transactions on Image Processing, Vol. 9, Pp. 1522-1531, September, 2000

102.Portilla J, Strela V, Wainwright $\mathrm{M}$ and Simoncelli E, Adaptive Wiener Denoising Using A Gaussian Scale Mixture Model. Proceedings International Conference On Image Processing, 2001

103.Figueiredo M.A.T and Nowak R.D, Wavelet-Based Image Estimation: An Empirical Bayes Approach Using Jeffrey's Noninformative Prior, IEEE Transactions on Image Processing, 10, Pp. 1322-1331, 2001

104.Hanssen A and Oigard T.A, The Normal Inverse Gaussian Distribution For Heavy-Tailed Processes, Proceedings IEEE EURASIP Workshop On Nonlinear Signal And Image Processing, 2001

105.Achim A, Bezerianos A and Tsakalides P, Novel Bayesian Multiscale Method For Speckle Removal In Medical Ultrasound Images', IEEE Transactions on Medical Imaging, 20, (5), Pp. 772783, 2001

106.Wainwright M., Simoncelli E and Willsky A, 'Random Cascade On Wavelet Trees And Their Use In Modeling Nutural Images' Applications of Computing and Harmonic Analysis, (2001), 11, Pp. 89-123, 2001

107.Portilla J, Strela V and Wainwright M.J and Simoncelli E.P, Image Denoising Using Gaussian Scale Mixtures In The Wavelet Domain, IEEE Transactions on Image Processing, 12, Pp. 1338-1351, 2003

108.Boubchir L and Fadili J, Bayesian Denoising In The Wavelet domain Using An Analytical Approximate Alpha-Stable Prior. Proceedings 17th International Conference In Pattern Recognition, August (2004), Cambridge, UK, 2004

109.J. M. Fadili And L. Boubchir, Analytical Form For A Bayesian Wavelet Estimator Of Images Using The Bessel K Form Densities, IEEE Transactions on Image Processing, Vol. 14, No. 2, Pp. 231240, February, 2005

110.Fadilim.J and Boubchir L, Analytical Formfor A Bayesianwavelet Estimator Of Images Using The Bessel K Form Densities, IEEE Transactions on Image Processing, 14, (2), Pp. 231-240, 2005

111.Boubchir L and Fadili J, Bayesian Denoising Based On The MAP Estimation In Wavelet-Domain Using Bessel $\mathrm{K}$ Form Prior. Proceedings 12th IEEE International Conference On Image Processing, September (2005), Genov, 2005

112.A. PiZurica and W. Philips, Estimating The Probability Of The Presence Of A Signal Of Interest In Multiresolution Single- And Multiband Image Denoising, IEEE Transactions on Image Processing, Vol. 15, Pp. 654-665, March, 2006.

113.Rabbani $\mathrm{H}$ and Vafadoost $\mathrm{M}$, Wavelet Based Image Denoising Based On A Mixture Of Laplace Distributions, Iranian Journal of Science and Technology, 30, (B6), Pp. 711-733, 2006

114.Bhuiyan M.I.H, Ahmad M.O and Swamy M.N.S, Wavelet-Based Despeckling Of Medical Ultrasound Images With The Symmetric Normal Inverse Gaussian Prior'. Proceedings 32nd International Conference On Acoustics, Speech, And Signal Processing, Hawai'I Convention Center In Honolulu, April (2007), USA, 2007

115.Rahman S.M.M, Ahmadm O and Swamy M.N.S, Bayesian wavelet based Image Denoising Using The Gauss-Hermite Expansion, IEEE Transactions on Image Processing, 17, (10), Pp. 1755-1771, 2008

116.Portilla J and Simoncelli E, Image Denoising Via Adjaustment Of Wavelet Coefficient Magnitude Correlation. Proceedings Seventh International Conference On Image Processing, 2000

117.Li X., Orchard M, Spatially Adaptive Denoising Under Overcomplete Expansion. Proceedings IEEE International Conference On Image Processing, (2000), Pp. 300-303, 2000

118.Fan G and Xia X, Image Denoising Using A Local Contextual Hidden Markov Model In The Wavelet Domain, IEEE Signal Processing Letters, (2001), 8, Pp. 125-128, 2001

119.Cai Z, Cheng T.H and Subramanian K.R, Efficient Waveletbased Image Denoising Algorithm, Electron. Letters, (2001), 37, (11), Pp. 683-684, 2001

120.Pizurica A, Philips W, Lemahieu I and Acheroy M, A Joint Interand Intrascale Statistical Model For Bayesian Wavelet Based Image Denoising, IEEE Transactions on Image Processing, 11, (5), Pp. 545-557, 2002

121.Sendur L and Selesnick I.W, Bivariate Shrinkage With Local Variance Estimation, IEEE Signal Processing Letters, (2002), 9, (12), Pp. 438-441, 2002

122.Zhan C.Q and Karam L.J, Wavelet-Based Adaptive Image Denoising With Edge Preservation. Proceedings IEEE International Conference On Image Processing, (2003), Vol. 1, Pp. 97-100, 2003
123.Kazubek M, Wavelet Domain Image Denoising By Thresholding And Wiener Filtering, IEEE Signal Processing Letters, (2003), 10, (11), Pp. 324-326, 2003

124.Eom I.K and Kim Y.S, Wavelet-Based Denoising With Nearly Arbitrarily Shaped Windows, IEEE Signal Processing Letters, (2004), 11, (12), Pp. 937-940, 2004

125.G. Fan And X. Xia, Image Denoising Using Local Contextual Hidden Markov Model In The Wavelet Domain, IEEE Signal Processing Letters, May (2001) Vol. 8, Pp. 125-128, 2001

126.M. J. Wainwright, E. P. Simoncelli, and A. S. Willsky, Random Cascades On Wavelet Trees And Their Use In Modeling And Analyzing Natural Images, Applications of Computing and Harmon. Analysis, Vol. 11, No. 1, Pp. 89-123, June, 2001

127.E. P. Simoncelli and B. A. Olshausen, Natural Image Statistics And Neural Representation, Annual Review of Neuroscience, Vol. 24, Pp. 1193-1216, 2001

128.J. Portilla, J. A. Guerrero-Colon, D. Van De Ville, V. Goyal, M. Papadakis and Eds, Image Restoration Using Adaptive Gaussian Scale Mixtures In Overcomplete Pyramids, In SPIE-Wavelets XII, September (2007), Vol. 6701, 2007

129.B. Goossens, A. Pi $`$ Zurica and W. Philips, Removal Of Correlated Noise By Modeling Spatial Correlations And Interscale Dependencies In The Complex Wavelet Domain, In Proceedings IEEE International Conference Image Processing, September (2007), Pp. 317-320, San Antonio, TX, 2007

130.Bart Goossen, Aleksandra Pi ${ }^{`}$ Zurica, and Wilfried Philips, Image Denoising Using Mixtures Of Projected Gaussian Scale Mixtures, IEEE Transactions On Image Processing, Vol. 18, No. 8, August, 2009

131.[131] David K. Hammond and Eero P. Simoncelli, Image Modeling And Denoising With Orientation-Adapted Gaussian Scale Mixtures IEEE Transactions On Image Processing, Vol. 17, No. 11, November, 2008

132.Jose A. Guerrero-Colón, Luis Mancera, and Javier Portilla, Image Restoration Using Space-Variant Gaussian Scale Mixtures In Overcomplete Pyramids, IEEE Transactions On Image Processing, Vol. 17, No. 1, January, 2008

133.Shubhankar Ray and Bani K. Mallick, A Bayesian Transformation Model For Wavelet Shrinkage, IEEE Transactions On Image Processing, Vol. 12, No. 12, December, 2003

134.Junmei Zhong, and Ruola Ning, Image Denoising Based On Wavelets And Multifractals For Singularity Detection, IEEE Transactions On Image Processing, Vol. 14, No. 10, October, 2005

135.Tamer Rabie, Robust Estimation Approach For Blind Denoising, IEEE Transactions On Image Processing, Vol. 14, No. 11, November, 2005

136.Onur G. Guleryuz, Nonlinear Approximation Based Image Recovery Using Adaptive Sparse Reconstructions And Iterated Denoising - Part Ii: Adaptive Algorithms, IEEE Transactions On Image Processing, Vol. 15, No. 3, March, 2006

137.Onur G. Guleryuz, Weighted Averaging For Denoising With Overcomplete Dictionaries, IEEE Transactions On Image Processing, Vol. 16, No. 12, December, 2007

138. Mark Miller, Image Denoising Using Derotated Complex Wavelet Coefficients, IEEE Transactions On Image Processing, Vol. 17, No. 9, September, 2008

139.Vincent Doré and Mohamed Cheriet, Robust Nl-Means Filter With Optimal Pixel-Wise Smoothing Parameter For Statistical Image Denoising, IEEE Transactions On Signal Processing, Vol. 57, No. 5, May, 2009

140.A. P.Witkin, Scale-space filtering," in Proceedings 8th International Joint Conference Artificial Intelligence, (1983), pp. 1019-1022, 1983

141.Pietro Perona and Jitendra Malik, Scale-Space and Edge Detection Using Anisotropic Diffusion, IEEE Transactions On Pattern Analysis And Machine Intelligence, Vol. 12. No. 7. July, 1990

142.F. Catté, P.-L. Lions, J.-M. Morel, and T. Coll, Image selective smoothing and edge-detection by nonlinear diffusion, SIAM J. Numerical Analysis, vol. 29, pp. 182-193, 1992

143.Y. You and M. Kaveh, Fourth-order partial differential equations for noise removal, IEEE Transactions on Image Processing, vol. 9, no. 10, pp. 1723-1730, October , 2000

144.M. J. Black, G. Sapiro, D. H. Marimont, and D. Heeger, Robust anisotropic diffusion, IEEE Transactions on Image Processing, vol. 7, no. 3, pp. 421-432, March 1998

145.Y. Chen, C. Barcelos, and B. Mair, Smoothing And Edge Detection By Time-Varying Coupled Nonlinear Diffusion Equations, Computer Vision and Image Understanding, vol. 82, pp. 85-100, mon1 
146.G. Sapiro and D. L. Ringach, Anisotropic Diffusion Of Multivalued Images With Applications To Color Filtering, IEEE Transactions on Image Processing, vol. 5, no. 11, pp. 1582-1586, November , 1996

147.D. Tschumperlé and R. Deriche, Vector-valued image regularization with PDEs: A common framework for different application, IEEE Transactions on Pattern Analysis and Machine Intelligence, vol. 27, no. 4, pp. 506-517, April, 2005

148.Karl Krissian, Carl-Fredrik Westin,Ron Kikinis, and Kirby G. Vosburgh, Oriented Speckle Reducing Anisotropic Diffusion, IEEE Transactions On Image Processing, Vol. 16, No. 5, May, 2007

149.Yi Wang, Liangpei Zhang, and Pingxiang Li, Local VarianceControlled Forward-and-Backward Diffusion for Image Enhancement and Noise Reduction. IEEE Transactions On Image Processing, Vol. 16, No. 7, July, 2007

150.L. Rudin, S. Osher, and E. Fatemi, Nonlinear Total Variation Based Noise Removal Algorithm, Physics. D, vol. 60, pp. 259-268, 1992

151.M. Nikolova, Minimizers of cost-functions involving non smooth data-fidelity terms, application to the processing of outliers, SIAM Journal of Numerical Analysis, vol. 40, no. 3, pp. 965-994, 2002

152.M. Nikolova, A variational approach to remove outliers and impulse noise, Journal of Mathematical Image and Visualization, vol. 20, pp. 99-120, 2004

153.Aria Abubakar Peter M. van den Berg, Tarek M. Habashy and Henning Braunisch, A Multiplicative Regularization Approach for Deblurring Problems, IEEE Transactions On Image Processing, Vol. 13, No. 11, November, 2004

154.Guy Gilboa, Nir Sochen, and Yehoshua Y. Zeevi, Variational Denoising of Partly Textured Images by Spatially Varying Constraints, IEEE Transactions On Image Processing, Vol. 15, No. 8, August, 2006

155.Wei-Wei Wang, Peng-Lang Shui, and Xiang-Chu Feng, Variational Models for Fusion and Denoising of Multifocus, Images IEEE Signal Processing Letters, Vol. 15, 2008

156.R. Chartrand V. Staneva, Total variation regularisation of images corrupted by non-Gaussian noise using a quasi-Newton method, IET Image Processing, Vol. 2, No. 6, pp. 295-303, 2008

157.Egil Bae, Juan Shi, and Xue-Cheng Tai, Graph Cuts for Curvature Based Image Denoising, IEEE Transactions On Image Processing, Vol. 20, No. 5, May, 2011

158.Jian $\mathrm{Wu}$ and Chen Tang, PDE-Based Random-Valued Impulse Noise Removal Based on New Class of Controlling Functions, IEEE Transactions On Image Processing, Vol. 20, No. 9, September, 2011

159. Wangmeng Zuo and Zhouchen Lin, A Generalized Accelerated Proximal Gradient Approach for Total-Variation-Based Image Restoration, IEEE Transactions On Image Processing, Vol. 20, No. 10, October, 2011

160.Guy Gilboa, Nir Sochen and Yehoshua Y. Zeevi, Image Enhancement and Denoising by Complex Diffusion Processes, IEEE Transactions On Pattern Analysis And Machine Intelligence, Vol. 26, No. 8, August, 2004

161.F. Barbaresco, Calcul des Variations et Analyse Spectrale: Equations de Fourier et de Burgers pour Modeles Autoregressifs Regularises, Traitement du Signal, vol. 17, nos. 5/6, 2000

162.R. Kimmel, R. Malladi, and N. Sochen, Images as Embedding Maps and Minimal Surfaces: Movies, Color, Texture, and Volumetric Medical Imaging International Journal of Computer Vision, vol. 39, no. 2, pp. 111-129, September, 2000

163.F. Russo and G. Ramponi, Fuzzy systems in instrumentation: fuzzy signal processing, IEEE Transactions on Instrumental Measurements, vol. 45, pp. 683-689, April, 1996

164.F. Russo, Recent advances in fuzzy techniques for image enhancement, IEEE Transactions on Instrumental Measurements, vol. 47, pp. 1428-1434, December, 1998

165.Y. Choi and R. Krishinapuran, A robust approach to image enhancement based on fuzzy logic, IEEE Transactions on Image Processing, vol. 6, pp. 808-825, June, 1997

166.F. Russo and G. Ramponi, Nonlinear fuzzy operators for image processing, Signal Processing, vol. 38, pp. 429-440, August, 1994

167.D. Zhang and Z.Wang, Impulse noise detection and removal using fuzzy techniques, Electronic Letters, vol. 33, no. 5, pp. 378-379, February, 1997

168.H. Qin and S. X. Yang, Nonlinear noise cancellation for image with adaptive neuro-fuzzy inference systems, Electronic Letters, April (2005) vol. 41, no. 8, pp. 474-475, 2005

169.E. Bes, dok, P. Civicioglu, and M. Alci, Using an adaptive neurofuzzy inference system-based interpolant for impulsive noise suppression from highly distorted images, Fuzzy Sets System, vol. 150 , no. 3, pp. 525-543, March, 2005

170.M. E. Y"uksel and E. Bes, dok, A simple neuro-fuzzy impulse detector for efficient blur reduction of impulse noise removal operators for digital images, IEEE Transactions on Fuzzy System, vol. 12 , no. 6, pp. 854-865, December, 2004

171.C. S. Lee, S. M. Guo, and C. Y. Hsu, Genetic-based fuzzy image filter and its application to image processing, IEEE Transactions on System, Man Cybern., B, Cybern., vol. 35, no. 4, pp. 694-711, August, 2005

172.M. E. Y"uksel, "A hybrid neuro-fuzzy filter for edge preserving restoration of images corrupted by impulse noise," IEEE Transactions on Image Processing, vol. 15, no. 4, pp. 928-936, April, 2006

173.S. Schulte, M. Nachtegael,V. DeWitte, D.Van derWeken, and E. E.Kerre, A fuzzy impulse noise detection and reduction method, IEEE Transactions on Image Processing, vol. 15, no. 5, pp. 11531162, May, 2006

174. Y. Nie and K. E. Barner, "Fuzzy rank LUM filters," IEEE Transactions on Image Processing, vol. 15, no. 12, pp. 3636-3654, December, 2006

175.S. Schulte, V. De Witte, M. Nachtegael, D. Van der Weken, and E. E. Kerre, Fuzzy random impulse noise reduction method, Fuzzy Sets System, vol. 158, no. 3, pp. 270-283, February, 2007

176.P. Civicioglu, Using uncorrupted neighborhoods of the pixels for impulsive noise suppression with ANFIS, IEEE Transactions on Image Processing, vol. 16, no. 3, pp. 759-773, March, 2007

177.[177] Sheng-Fu Liang, Shih-Mao Lu, Jyh-Yeong Chang and ChinTeng (CT) Lin, A Novel Two-Stage Impulse Noise Removal Technique Based on Neural Networks and Fuzzy Decision, IEEE Transactions On Fuzzy Systems, Vol. 16, No. 4, August, 2008

178.[178] Kenny Kal Vin Toh and Nor Ashidi Mat Isa, Noise Adaptive Fuzzy Switching Median Filter for Salt-and-Pepper Noise Reduction, IEEE Signal Processing Letters, Vol. 17, No. 3, March, 2010

179.Kenny Kal Vin Toh and Nor Ashidi Mat Isa, "Cluster-Based Adaptive Fuzzy Switching Median Filter for Universal Impulse Noise Reduction" IEEE Transactions On Consumer Electronics, Vol. 56, No. 4, November, 2010

180.L. Gu and Y. Q. Zhang, Web shopping expert using new interval type-2 fuzzy reasoning, Soft Computing, vol. 11, no. 8, pp. 741751,2007

181.C. Lynch, H. Hagras, and V. Callaghan, Embedded type-2 FLC for realtime speed control of marine and traction diesel engines, in Proceedings FUZZ IEEE (2005), Reno, AZ, pp. 347-352, 2005

182.L. Astudillo, O. Castillo, P. Melin, A. Alanis, J. Soria, and L. Aguilar, Intelligent control of an autonomous mobile robot using type-2 fuzzy logic, Journal of Engineering Letters, vol. 13, no. 2, pp. 93-97, 2006

183.H. Hagras, A hierarchical type-2 fuzzy logic control architecture for autonomous mobile robots, IEEE Transactions on Fuzzy System, vol. 12, no. 4, pp. 524-539, August, 2004

184.J. Debayle and J. C. Pinoli, General Adaptive Neighborhood Image Processing - Part I: Introduction and Theoretical Aspects, Journal of Mathematical Imaging and Vision, 25(2):245--266, September, 2006

185.N. Bouaynaya, M. Charif-Chefchaouni and D. Schonfeld, Theoretical foundations of spatially-variant mathematical morphology - Part I: Binary images, IEEE Transactions on Pattern Analysis and Machine Intelligence, vol. 30, pp. 823- 836, 2008

186.N. Bouaynaya and D. Schonfeld, Theoretical foundations of spatially-variant mathematical morphology - Part II: Graylevel images, IEEE Transactions on Pattern Analysis and Machine Intelligence, vol. 30, pp. 837-850, 2008

187. Soille. P and Talbot. H, Directional morphological filtering, IEEE Transactions on Pattern. Analysis Machine Intelligence, 23, (11), pp. 1313-1329, 2001

188.C. Vachie and F. Meyer. News from viscous Land. International Symposium on Mathematical Morphology, pages 189--200, October (2007), Rio de Janeiro, Brazil, 2007

189.Hamid, M.S, Harvey, N.R and Marshall. S, Genetic algorithm optimization of multidimensional grayscale soft morphological filters with applications in film archive restoration, IEEE Transactions on Circuits System Video Technology, (2003), 13, (5), pp. 406-416, 2003

190.Ji, T.Y, Lu, Z, Wu and Q.H, Optimal soft morphological filter for periodic noise removal using a particle swarm optimiser with passive congregation, Signal Processing, (2007), 87, (11), pp. 7709-7800 วח07 
191.Tao, L., Yang-yu, F, Lian-bing and H, Fast lane recognition based on morphological multi-structure element model, Optoelectronic Letters, (2009), 5, (4), pp. 304-308, 2009

192.Nishida, S, Nakamura, M, Ikeda, A, Shibasaki and H, Signal separation of background EEG and spike by using morphological filter, Medical Engineering and Physics, (1999), 21, (9), pp. 601608,1999

193.Xu, J, A generalized discrete morphological skeleton transform with multiple structuring elements for the extraction of structural shape components, IEEE Transactions on Image Processing, 12, (12), pp. 1677-1686, 2003

194.T. Lei and Y.-Y. Fan, Noise gradient reduction based on morphological dual operators, IET Image Processing, Vol. 5, Iss. 1, pp. 1-17, 2011 